\title{
Design of the Control System about Central Signals in Electric Vehicle
}

\author{
Qianqian Zhang, Yan Wang, Tianming Yin
}

Electrical Engineering School, Beijing Jiaotong University, Beijing, China.

Email: zhang.q_0401@126.com, yanwang@mail.bjtu.edu.cn

Received December $1^{\text {st }}, 2009$; revised January $6^{\text {th }}, 2010$; accepted January $10^{\text {th }}, 2010$.

\begin{abstract}
Based on the development demand of electric vehicles, this paper studies the communication network in electric vehicles based on CAN bus after analyzing the LCD (liquid crystal display) in electric vehicles and the status quo of research and development trend about communication network. This system uses TMS320LF2407 DSP as the controller of the control system about central signals. It has also developed the LCD system based on CAN bus and the experiment platform which is applied pure electric vehicles.
\end{abstract}

Keywords: CAN Bus, DSP, Electric Vehicle, LCD System, SCI

\section{Introduction}

Field bus is one hot topic in the developing technology in the automatic field. It is praised as Regional Network of Computers in the automatic field. Its appearance provides powerful technical support for DCS (Distributed Control System) to realize real-time and reliable data communication between each node. CAN (Control Area Network) belong to the category of field bus. It was developed by Germany BOSCH Company in early 1980s to solve data interchange between numerous control and test instruments in vehicles. Because of its outstanding reliability, real-time and flexibility, CAN bus is approved and applied in the industry. And it became the international standard and industry standard formally in 1993 [1]. It also is praised as one of the most promising field buses. The application of bus technology with CAN as the representative in vehicles not only reduces the harness of the car but also increases the reliability of the car.

Motor meter is the alternation interface between the user and the vehicle. It can provide necessary information such as operating parameters, warning and mileage for drivers. Therefore, motor meter is the requisite part. However, traditional meter can only provide a small number of necessary data information for drivers. This is far from the requirement of new technology and high speed in modern vehicles. Motor meter should not only display information simply but also control all kinds of operation conditions of the vehicle through the monitoring of parameters in every part in the vehicle. With the development of modern electron technology, meter with multifunction, high accuracy, high sensitivity, direct and reliable reading and picture display has been applied in the vehicle gradually. Therefore, electronic instrument will replace conventional electromechanical instrument gradually.

This paper applies CAN bus technology in the control system of electric car and combines it with LCD system. It has realized to improve the performance of the control system in the electric vehicle.

\section{The Application of Can Bus in Electric Vehicles}

CAN bus which belongs to the category of field bus is a kind of serial communication network which supports distributed control or real-time control effectively.

Currently, many existing auto network standards have different emphasis about their functions. SAE traffic network committee classified the data transmission network into three kinds including A type, B type and C type for convenient study, design and application, design and application [2]. A type turns towards Low Speed Network controlled by sensors or actuators. The bit rate of data transmission usually is only $1 \sim 10 \mathrm{~kb} / \mathrm{s}$. They are mainly used to control the electric windows, seat adjust and lamplight illumination. B type turns toward the moderate speed network of data sharing between independent modules. The bit rate usually is $10 \sim 100 \mathrm{~kb} / \mathrm{s}$. They are mainly used in systems such as information centre of electronic vehicle, fault diagnosis, instrument display and air bag to reduce redundant sensors and other 
electronic units. C type turns toward the multipath transmission with high speed and real-time closed-loop control. The highest bit rate is $1 \mathrm{Mb} / \mathrm{s}$. They are mainly used in systems such as suspension control, traction control, advanced motor control and ABS to simplify the distributed control and the harness further. As so far, only CAN protocol satisfy the demand of $\mathrm{C}$ type in the auto control LAN.

\section{Design of the Vehicle Communication System Based on Can Bus}

\subsection{Schematic Diagram of the System}

This system is mainly made up of control modules, motor drive module, BMS module, service module and meter display module. TMS320LF2407 is the processor [3]. Because there is a controller of CAN, only one transceiver of CAN is needed. The information channel between each module will be realized through CAN bus. Except for the sending and receiving of instructions, the basic status information of vehicles is the requisite data obtained by most control modules. Control modules send data to the bus using broadcast mode. If all control units send data to the bus at the same time, it will cause data collision in the bus. Therefore, CAN bus protocol proposed the bus arbitration which used identifier to identify the priority of data. Table 1 presents data types that will be received or sent by ECU in electric vehicles and the sharing of other units about these information.

\subsection{CAN Bus Design}

\subsubsection{CAN Bus}

The gross structure of CAN bus shows as Figure 2. There are two resistances with the value of $120 \Omega$ in each side

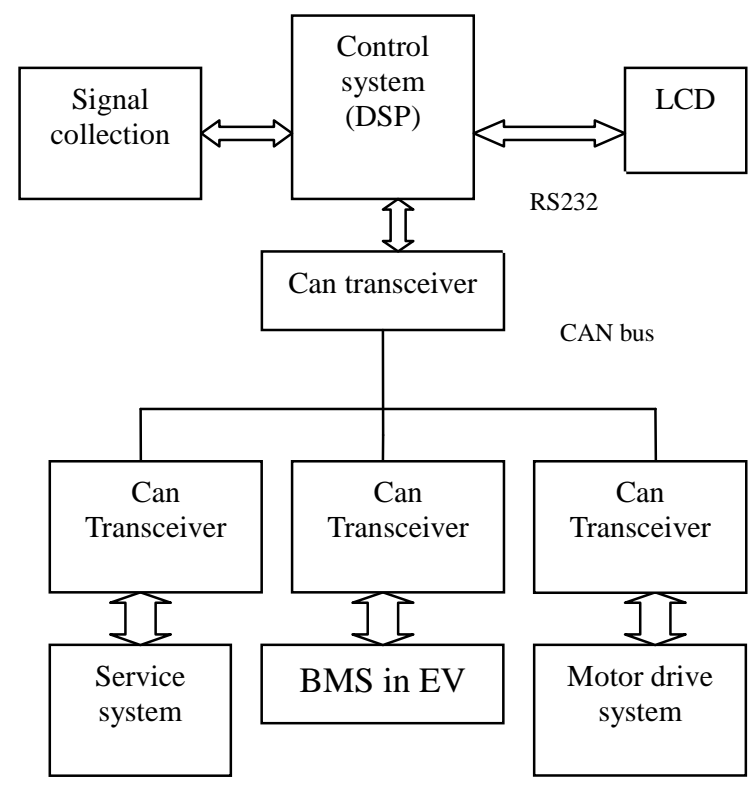

Figure 1. Schematic diagram
Table 1. Data types sent or received by ECU

\begin{tabular}{|c|c|c|c|c|c|}
\hline Signal type & $\begin{array}{c}\text { Cen- } \\
\text { tral } \\
\text { signal } \\
\text { control }\end{array}$ & $\begin{array}{l}\text { Motor } \\
\text { drive } \\
\text { control }\end{array}$ & $\begin{array}{c}\text { Battery } \\
\text { man- } \\
\text { age- } \\
\text { ment } \\
\text { module } \\
\end{array}$ & $\begin{array}{c}\text { Service } \\
\text { facilities } \\
\text { inside } \\
\text { cars }\end{array}$ & $\begin{array}{l}\text { LCD } \\
\text { unit }\end{array}$ \\
\hline Motor speed & $\mathrm{R} \& \mathrm{~T}$ & $\mathrm{~T}$ & & & $\mathrm{R}$ \\
\hline $\begin{array}{c}\text { Motor } \\
\text { temperature }\end{array}$ & $\mathrm{R} \& \mathrm{~T}$ & $\mathrm{~T}$ & & & $\mathrm{R}$ \\
\hline $\begin{array}{l}\text { Regenerative } \\
\text { braking }\end{array}$ & & In & & & \\
\hline Motor error & $\mathrm{R} \& \mathrm{~T}$ & $\mathrm{~T}$ & & & $\mathrm{R}$ \\
\hline $\begin{array}{l}\text { Forward } \\
\text { /reverse }\end{array}$ & $\mathrm{T}$ & $\mathrm{R}$ & & & \\
\hline Speed & $\mathrm{R} \& \mathrm{~T}$ & $\mathrm{~T}$ & & & $\mathrm{R}$ \\
\hline $\begin{array}{c}\text { Remaining } \\
\text { battery } \\
\text { power }\end{array}$ & $\mathrm{R} \& \mathrm{~T}$ & & $\mathrm{~T}$ & & $\mathrm{R}$ \\
\hline $\begin{array}{l}\text { Battery } \\
\text { charging } \\
\text { status }\end{array}$ & $\mathrm{R} \& \mathrm{~T}$ & & $\mathrm{~T}$ & & $\mathrm{R}$ \\
\hline $\begin{array}{l}\text { Temperature } \\
\text { inside cars }\end{array}$ & R\&T & & & $\mathrm{T}$ & $\mathrm{R}$ \\
\hline $\begin{array}{c}\text { LCD } \\
\text { switching }\end{array}$ & $\mathrm{T}$ & & & & $\mathrm{R}$ \\
\hline
\end{tabular}

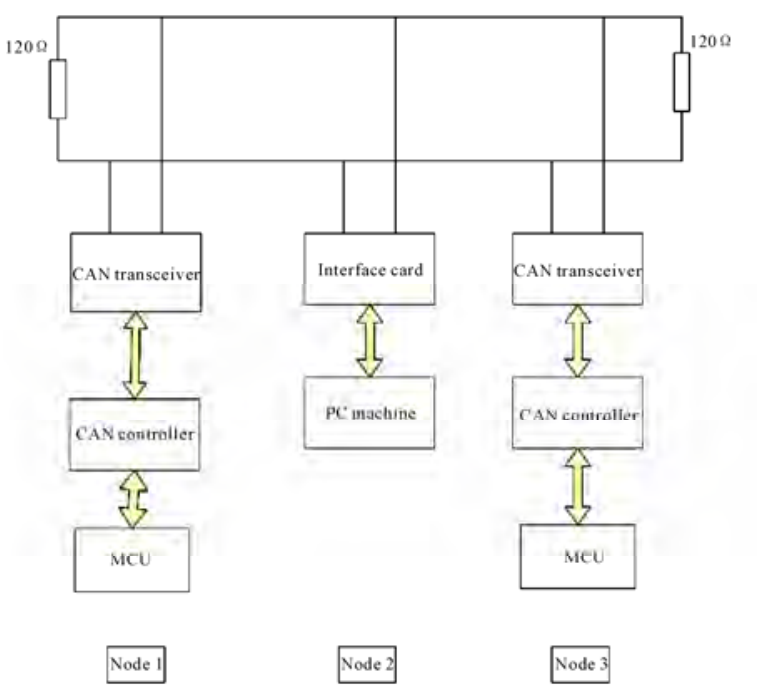

Figure 2. Structural diagram of CAN bus system

of the bus. They are resistances matching the bus. They can increase the stability and the capacity of resisting disturbance of bus transmission and reduce the error rate in the process of data transmission [4]. The node structure of CAN bus can be classified into two kinds: the first is to connect the CAN adapter with PC machine to realize the communication between the host computers and CAN bus; the other is made up of microcontroller, CAN controller and CAN actuator. It transmits data with CAN bus as one kind of node. In this system, CAN controller is SJA1000 produced by Philips as the send and receive buffer to realize the data transmission between the main 
controller and the bus [5]; CAN transceiver is PCA82C250 chip which is the interface of CAN controller and physical bus. It can provide differential send ability for the bus and differential receive ability for the CAN controller.

\subsubsection{CAN Bus Transceiver PCA82C250}

PCA82C250 is the interface of CAN protocol controller and physical bus. It can provide differential send ability and receive ability of the bus. Different operation modes can be chose through pin 8 (RS): high speed, slope control and stand by. In the operation mode of high speed, the output transistor of the transmitter conducts or cuts off as soon as possible. In this mode, no measure is needed to limit the rising and descending slope. UTP (unshielded twisted pair) or parallels can be used as the bus in the situation of lower speed or shorter bus. The rising and descending slope should be limited to reduce RF interference. And they can be controlled by the earth resistance in pin 8 . The operation mode of slope is used in this system. In the network made up of PCA82C250, when the input in the TXD side of PCA82C250 is high level, its output sides CANH and CANL are all in high-impedance state in which PCA82C250 has no influence on the whole network. Then, this node is in concession state; when the input of TXD is low level, CANH and CANL output high level and low level respectively. Then this node decides the nature of the transmission data in the whole network. The output level of RXD in PCA82C250 is consistent with the level of RXD which plays the dominating role in the network.

The circuit of CAN interface is shown as Figure 3.

In this figure, B0505LD is the Power Isolated Module which can isolate the power in the bus from the power supply of DSP and play the role of protection. In the auto environment, voltage often changes instantly. If there is protection measure, it is very possible that electronic devices can be destroyed by the instant change of the voltage. In order to suppress the instant change of voltage, capacitance can be added between CAN bus and the ground wire to filter out noise and make signals more stationary.

\section{Design of Color LCD}

\subsection{Main Display Function and Plan}

Comprehensive display interface: set the state of speed, mileage, alarm message, battery state, whole power of batteries, whole voltage of batteries and SOC [6].

Combined transformation interface: in order to display the information of electric motor and battery, both interface of motor state and interface battery will be set. They will display information of the two modules respectively in detail and give alarms.

Information display methods include analog display (change and trend), digital display (direct and correct), graphic symbol display (vivid) and text display (implication) [7].

Expected display interface of comprehensive information is shown as Figure 4: vehicle speed, motor speed, remaining power of batteries and fault information will be displayed in this interface. At the bottom of the screen, the interface will be switched to information interface of battery, electric motor and service facilities respectively.

\subsection{Program Design of Serial Communication of LCD Module}

LCD adopts standard RS232 communication interface and works in serial interface mode. The receiver and transmitter of SCI is double buffering. Each has its separate enabling and interruption flag bit. Both can work alone in the full-duplex mode at the same time. SCI uses even parity check, overtime detection and error frame detection to ensure correct transmission of data. Two external pins of SCI including SCITXD and SCIRXD can be used as common I/O when they are not used in the communication. The subprogram of serial communication is shown as Figure 5.

After disabling all interruptions and clearing interrupt

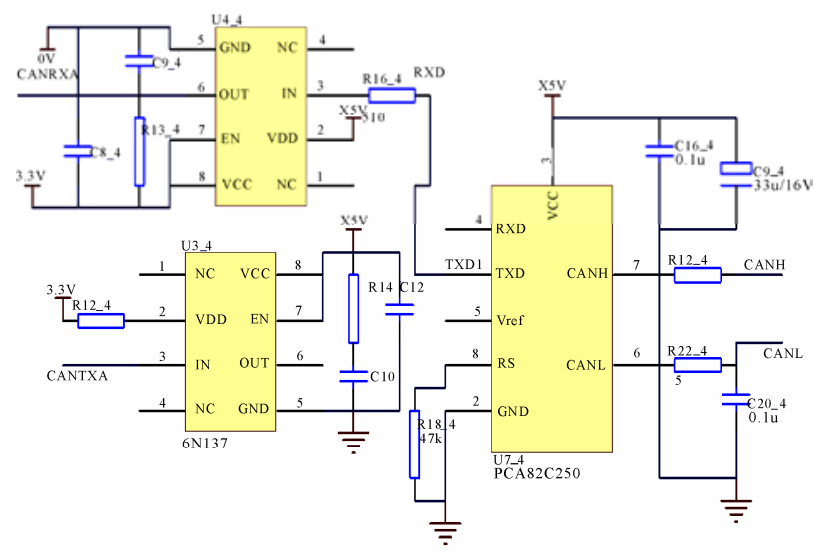

Figure 3. Interface circuit of TMS320LF2407

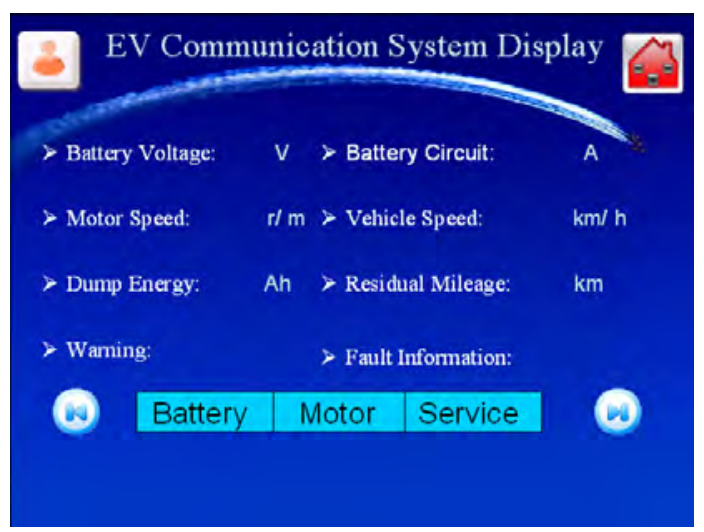

Figure 4. Display interface of comprehensive information 


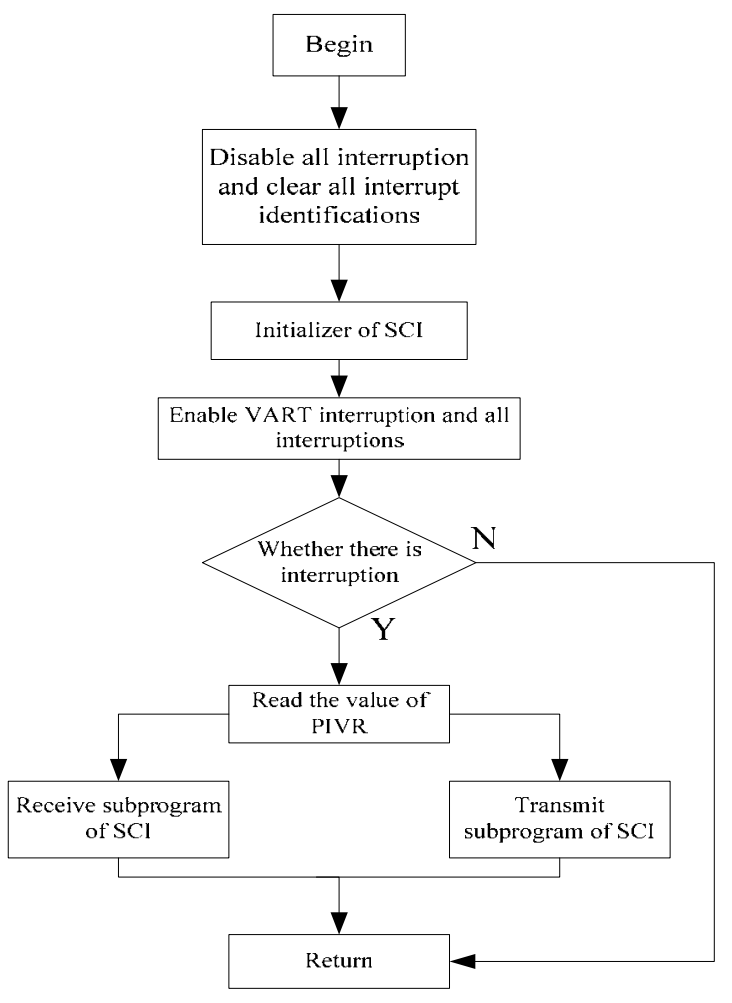

Figure 5. Flow chart of serial communication subprogram

identifications, the procedure will start the initializer of SCI which includes the setting of communication controller SCICCR of serial communication interface. For example, one stop bit, disable parity check, idle line and multiprocessor mode and 8 bits character. After the initializer, SCI interruption INT5 will be enabled. When the interruption is coming, judge the value of the register PIVR of the interrupt vector. If PIVR is 6 , then receiving interruption happened and receiving service program will be executed; if PIVR is 7, transmitting interruption happened and transmitting service program will be executed.

\section{Experiment Results}

The physical connection diagram on the test bench is shown as Figure 6 in which the upper is the LCD; the left is the BMS module; the right is the control system about central signals with DSP as the core chip; the UTP between the two modules is the CAN bus.

The node of the host computer is made up of CAN interface card, PC machine and monitoring software. Among them CAN interface card is one kind of unintelligent adapter of CAN developed by Beijing Run Quan scientific and technological company. It is a kind of unintelligent adapter connecting CAN communication protocol with PC machine. Besides, it can satisfy the requirement of high real-time of the network. Meters with CAN communication interface and controlling equipments in industrial field can be monitored through CAN card.
The data received by CAN bus can be displayed on the color LCD in real time. The color LCD can dynamically display information such as the speed, mileage, whole power of batteries, temperature of the battery, remaining power and battery state in real time. The result is shown in Figure 7.

To get the information of $\mathrm{EV}$ in detail, the system interface will be available by pressing button $\Rightarrow$, the display interface is shown as Figure 8.

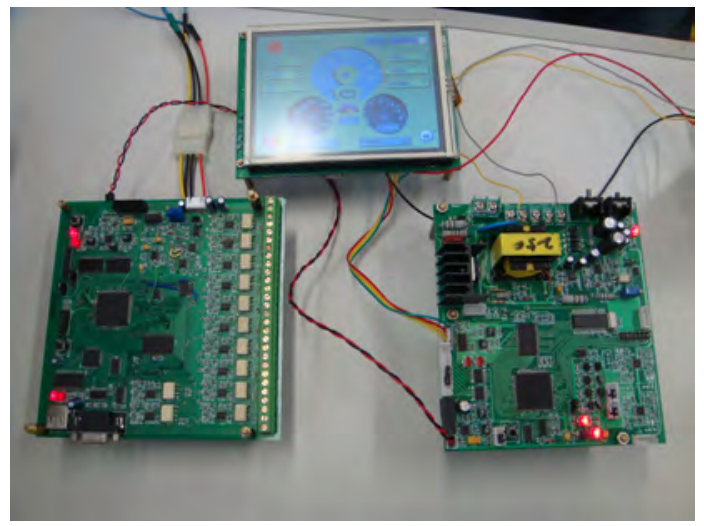

Figure 6. Test system connection

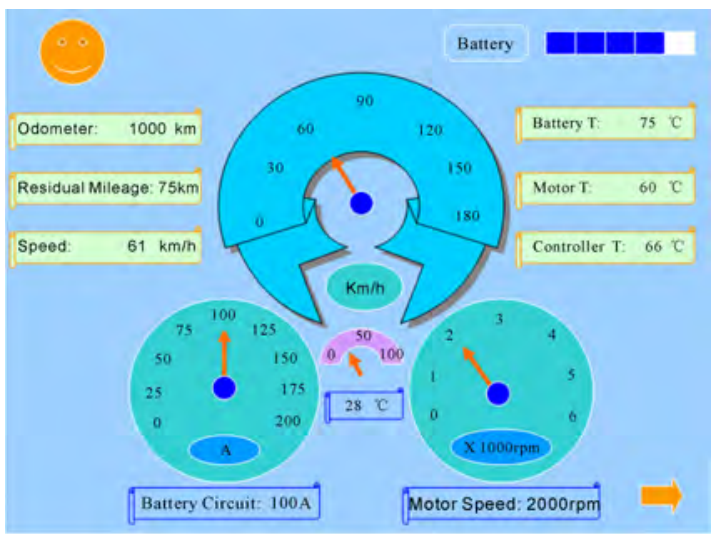

Figure 7. Information in the dashboard

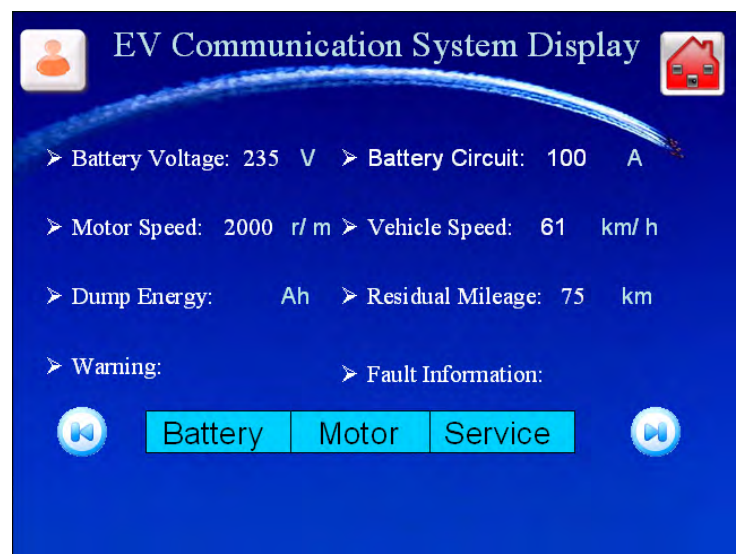

Figure 8. Liquid crystal display of communication system 
In this experiment, some analog signals are given to the controller (DSP) in the control system about central signals. Through SCI communication, they are all shown in the LCD successfully as shown in Figure 8.

The Dashboard interface will be available again by pressing button

The battery interface will be available by pressing button "Battery", the display interface is shown as Figure 9 and Figure 10.

As shown in Figure 9 and Figure 10, there are 20 batteries in EV. The monomer battery voltage can be got through A/D sampling. Because the battery current will not change frequently, the current information will be only shown in the dashboard interface. This is realized successfully shown as Figure 10. However, the dump power is unable to obtain currently. In the future, it can be obtained through some algorithms.

Similarly, the motor interface and service interface will be available by pressing button "Motor" and "Service" respectively. The display interface is shown as Figure 11 and Figure 12 respectively.

Similarly, the dashboard interface will be available again by pressing

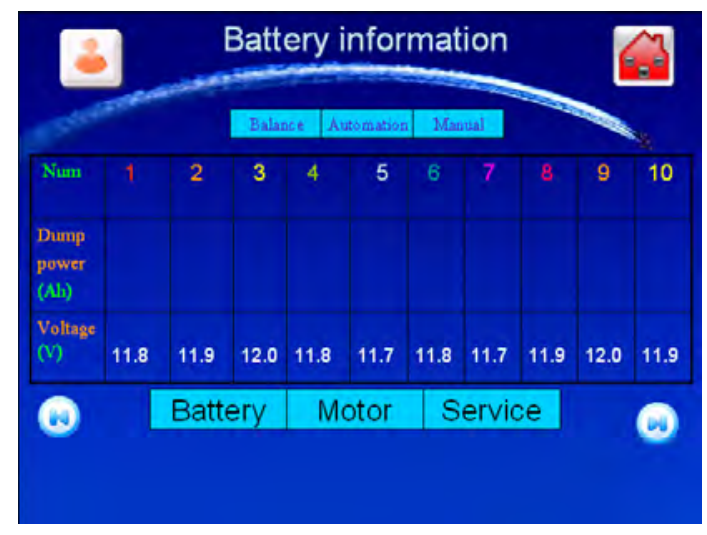

Figure 9. Battery information 1

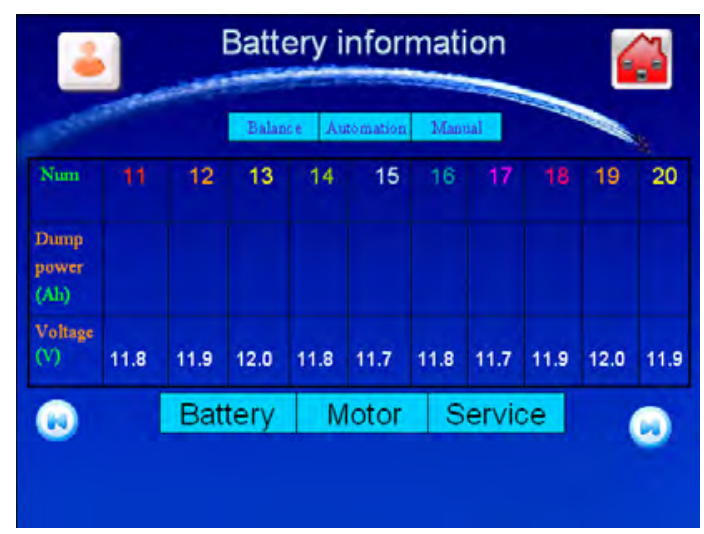

Figure 10. Battery information 2

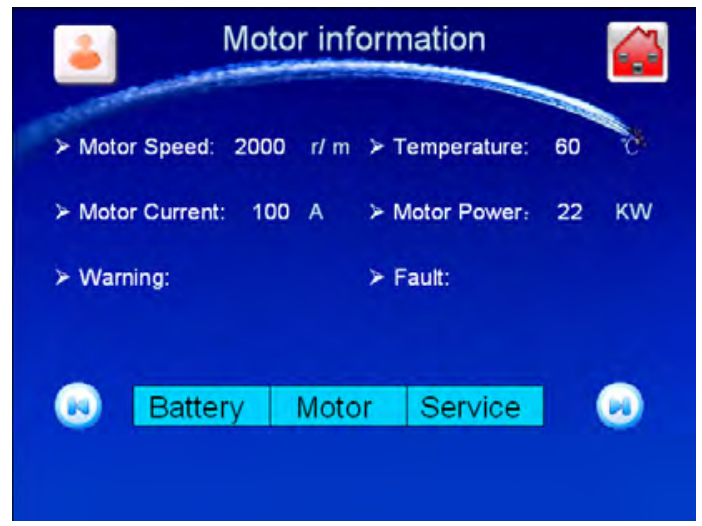

Figure 11. Motor information

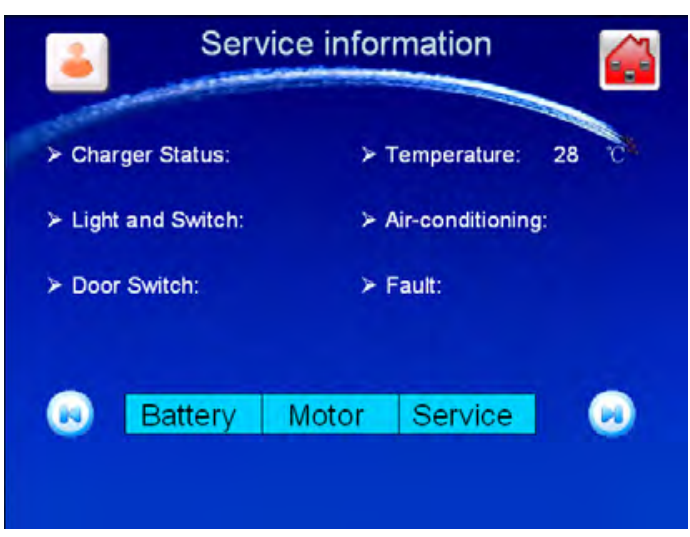

Figure 12. Service information

Currently, how to dispose some information about light and switch, charger status and door switch is still in the study.

\section{Conclusions}

This paper introduced the features of CAN bus and its application in electric vehicles and designed the nodes of control system in the whole electric vehicle based on CAN bus. Finally it displayed the state information of the vehicle using liquid crystal display.

This paper also did corresponding experiment design on the electronic control system in the whole vehicle. Besides, it record and analyzed the results of the experiment. The results proved that this system was stable. The output waveform is stable of each node in CAN bus and the communication with electric motor and battery is normal. The information can be displayed on the liquid crystal screen. These satisfy the demands of the experiment.

\section{REFERENCES}

[1] SAE J1939-13, “Off-board diagnostic connector,” Society of Automotive Engineers, USA, July 1999.

[2] SAE J1939-31, “(R) Network layer,” Society of Automotive Engineers, USA, December 1994. 
[3] R. S. Hourani, “A performance analysis framework for the design of DSP systems," Dissertation Abstracts International, Vol. 69, No. 4, p. 2517, 2008.

[4] G. P. Liu, “Anticipating system-level electromagnetic interference using numerical methods and measurement techniques,” Dissertation Abstracts International, Vol. 65, No. 8, p. 4195, 2004.

[5] H. Huang, "Electrical two speed transmission and ad- vanced control of electric vehicles," Dissertation Abstracts International, Vol. 60, No. 7, p. 3455, 1998.

[6] F. Fang, "A solid state relay battery management system for series connected batteries," Dissertation Abstracts International, Vol. 63, No. 8, p. 3640, 2002.

[7] F. S. Zhou, "Reflective and transflective liquid crystal displays," Dissertation Abstracts International, Vol. 67, No. 1, p. 341, 2005. 